\title{
Dynamic In Vivo Imaging of Post-Implantation Mammalian Embryos Using Whole Embryo Culture
}

E. A. V. Jones ${ }^{1}$, D. Crotty², P. M. Kulesa ${ }^{2}$, C.W. Waters ${ }^{2}$, M.H. Baron ${ }^{3}$, S.E. Fraser', M.E. Dickinson ${ }^{2}$

${ }^{1}$ Department of Chemical Engineering, California Institute of Technology, 1200 E California Blvd., Pasadena, CA, 91125, USA

${ }^{2}$ Department of Biology, California Institute of Technology, 1200 E California Blvd., Pasadena, CA, 91125, USA

${ }^{3}$ Mount Sinai School of Medicine of NYU, Box 1079, 1425 Madison Avenue 11-70B, New York, NY, 10029

Corresponding Author:

Mary E Dickinson

MC 139-74

1200 E California Blvd

Pasadena, CA, 91125

Tel : (626)395-2054

Fax: (626) 584-9527

Email: maryd@gg.caltech.edu 


\section{Abstract}

Due to the internal nature of mammalian development, much of the research performed is of a static nature and depends on interpolation between stages of development. This approach cannot explore the dynamic interactions that are essential for normal development. While roller culture overcomes the problem of inaccessibility of the embryo, the constant motion of the medium and embryos makes it impossible to observe and record development. We have developed a static mammalian culture system for imaging development of the mouse embryo. Using this technique, it is possible to sustain normal development for periods of $18-24$ hours. The success of the culture has been evaluated based on the rate of embryo turning, heart rate, somite addition, and several gross morphological features. When this technique is combined with fluorescent markers, it is possible to follow the development of specific tissues or the movement of cells. To highlight some of the strengths of this approach, we present time-lapse movies of embryonic turning, somite addition, closure of the neural tube and fluorescent imaging of blood circulation in the yolk sac and embryo. 


\section{Introduction}

Through sophisticated genetic manipulation and examination of naturally occurring mutations, hundreds of genes have been identified that play key roles in early mouse development. As more is learned about genetic hierarchies that control morphogenesis, additional questions arise concerning how cells behave in response to both molecular and environmental cues. Mutations can cause a multitude of results, from absence of certain structures to malformations of embryonic tissues. Defects can result from changes in cell fate, apoptosis of progenitor cells, lack of differentiation of progenitors or from incorrect cell migration. Thus, it is important to understand how cells interact in normal development and how mutations change these interactions, resulting in abnormal development.

Because mouse embryos develop in utero, most of what is known about dynamic events such as gastrulation and neural tube closure has been deduced from static images of embryos at successive stages of development. Dynamic cellular interactions have been studied in other, more accessible systems, such as Xenopus, zebrafish or avian embryos. In these systems, labeled cells can be visualized over time using time-lapse light microscopy to determine migration trajectories and behaviors, to observe cell-cell contacts, as well as to determine cell fate relationships. Such approaches have been limited in the early mouse embryo because culture conditions suitable for time-lapse microscopy have not been established. While embryos up to late 7.5 days post coitum $(\mathrm{dpc})$ can be grown in static cultures suitable for imaging, older embryos, from 8.5-dpc onwards, have traditionally been cultured in roller flasks (Tam, 1998). It has generally been thought that the gas exchange provided by roller culture was essential for normal embryonic development.

Here we present a robust, static culture method for embryos isolated from $8.5-\mathrm{dpc}$ to $9.5-\mathrm{dpc}$. We show that by controlling $\mathrm{pH}$, gas exchange and evaporation, embryos at these stages can JONES 
be grown in a culture dish placed directly on the microscope stage, enabling time-lapse analysis of their development. Normal development is sustained for at least 18-24 hours. Several critical parameters of the growth media and culture environment were tested and are described here. Time-lapse movies of embryos in culture are provided, illustrating the success of the technique.

\section{Results}

The goal of these experiments is to provide a method for observation of the development of the post-implantation mouse embryos. For meaningful results, in vitro development must recapitulate in utero development. Because roller culture is an accepted means of culturing mouse embryos, the initial goal of this research was to develop growth conditions comparable to roller culture. Success was judged by several factors. For 8.5 -dpc embryos, the ability of the embryos to turn was recorded and compared with in vivo and roller culture results. The embryos were also examined for normal head fold closure, heart looping, vascular development and heart rates, as compared to in vivo growth. Embryos were staged using a system that consisted of 22 stages defined by images of freshly dissected 8.5-dpc embryos (described in Table 1, images not shown). A more traditional staging system, such as the one proposed by Brown \& Fabro (Brown \& Fabro, 1981) was not used since the system requires the removal of the yolk sac to evaluate many features. Since 8.5-dpc embryos were cultured with the yolk sac intact, it would not have been possible to evaluate the initial age. For 9.5-dpc embryos, rate of somite addition and heart rate were used as the main criteria for normal development. Since somite addition relates linearly to embryonic growth, we used this criterion to assess extent of embryonic growth (Brown \& Fabro, 1981). Heart rates were measured throughout the culture period. 


\section{Culture chambers and temperature control of embryos on the microscope}

stage

Embryos were cultured in Labtek chambers consisting of two plastic culture wells attached to a coverslip via a silicon gasket. Embryos were imaged from below, through the coverslip, with an inverted microscope. Embryos were cultured three per chamber. In order to keep the medium static, yet enable aeration and $\mathrm{pH}$ regulation, the inside of the culture chamber was supplied continuously with heated humidified gas. The whole system, including the microscope optics, was kept at constant temperature using a chicken egg incubator heater enclosed within a mylarinsulated box (Figure 1; described in detail in the Materials and Methods section).

\section{Culture media and additives}

The first culture condition that was optimized was the composition of the medium. The literature on roller culture highlighted the need for high quality rat serum, as well as a wide range of possible media additives (Copp \& Cockroft, 1990). These included vitamins (Martin \& Cockroft, 1999), glucose (Tam, 1998) (Martin \& Cockroft, 1999), transferrin (Katoh, et al., 1998), inositol (Tam, 1998), albumin (Katoh, et al., 1998) and HEPES buffer. Complete DMEM/F12 medium was chosen as a starting point for the medium formulation because it already contained vitamins, glucose and inositol.

As with other published culture methods, a high concentration of rat serum in the medium was found to be essential (Copp \& Cockroft, 1990). Commercial sources of rat serum were tested and results compared to those obtained from serum freshly prepared in our own lab (see Table 2). Commercial sources of rat serum were adequate for normal culture of 9.5-dpc embryos, 
resulting in the development of embryos with normal heart rates and somite addition rates (Table 2). After optimization with the 9.5-dpc embryos, the best of these commercial sera, from Seracare, was then tested for culture of 8.5-dpc embryos. These embryos never completed the turning process in the commercial sera. Therefore, our homemade serum was used for all subsequent studies.

We tested HEPES buffer solution as a means to regulate $\mathrm{pH}$ fluctuations in the medium. Two different concentrations (5 and $10 \mathrm{mM}$ ) were tested. For both concentrations, similar final $\mathrm{pH}$ values were measured at the end of the culture period (average $\mathrm{pH}$ for $5 \mathrm{mM}$ HEPES was 7.5 $( \pm 0.3, n=34) ; 7.6( \pm 0.2, n=20)$ for $10 \mathrm{mM}$ HEPES $)$. The desired $\mathrm{pH}$ should be between 7.0 and 7.2. As such, a greater level of control over the $\mathrm{pH}$ is necessary and will be discussed later. A concentration of $10 \mathrm{mM}$ HEPES was used for all subsequent cultures.

Transferrin is an iron storage protein produced both by maternal tissues and by the yolk sac (Janzen, et al., 1982). Based on previously reported uses of transferrin in whole embryo culture (Bulic-Jakus, et al., 1999), a concentration of $10 \mu \mathrm{g} / \mathrm{mL}$ was tested. Embryos cultured in the presence of $10 \mu \mathrm{g} / \mathrm{mL}$ of transferrin had significantly lower heart rates (average of 40 beats per minute or bpm, $n=12$ for $8.5-d p c, n=6$ for $9.5-d p c)$ by the end of the culture period. Therefore, transferrin was omitted from subsequent cultures.

Albumin is an essential blood component and is also known to deteriorate quickly in preparations of rat serum (Katoh, et al., 1998). We therefore tested whether the addition of albumin could improve the culture. Albumin collected from chicken egg whites was added at a range of concentrations, from $50 \mu \mathrm{L}$ to $250 \mu \mathrm{L}$ per $\mathrm{mL}$ of media. The embryos cultured under these conditions had no heartbeats after 24 hrs of culture $(n=12)$, even though temperature and $\mathrm{pH}$ were kept constant for the entire culture period. 
Based on these experiments the preferred medium composition consists of $50 \%$ rat serum, $50 \%$ DMEM/F12, supplemented with HEPES and Pen-Strep (see Materials and Methods).

\section{Aeration of culture chamber}

Because roller culture offers better gas exchange than static culture, the effects of aeration and diffusion were investigated. Even with proper medium formulation, static cultures were still not as successful as cultures in roller flasks. pH levels were too high by the end of culture (average of 7.6 compared with a desired 7.2 , as previously reported) and the percentage of 8.5 -dpc embryos that turned was also low $(11 \%, n=38)$.

It has previously been reported that the yolk sac becomes a barrier to nutrient transport after the embryo has turned (Eto \& Osumi-Yamashita, 1995). We tested whether the yolk sac was hindering nutrient transport by culturing 9.5-dpc embryos with and without yolk sacs $(n=12$, $\mathrm{n}=70$ respectively), and found that the presence of the yolk sac impedes development. 9.5-dpc embryos cultured with yolk sacs did not survive 24 hours in culture (as defined by the absence of a heartbeat). Therefore, the yolk sac was removed for 9.5-dpc embryos, while younger embryos were cultured with intact yolk sacs.

The effects of gas flow rate were also investigated since gas transfer rates are lower in static culture. Rapid evaporation from the medium was observed at high flow rates. However, even at low flow rates, some evaporation occurred and was found to be detrimental to the embryonic development, suggesting that embryos are sensitive to even small changes in the concentration of medium components or secreted waste products. Evaporation causes yolk sacs of 8.5-dpc embryos to become wrinkled and caused the circulation in the yolk sac to stop. Under these conditions, the embryos did not complete the turning process. Evaporation is apparently not a 
problem in roller culture where the embryo chambers are maintained at higher backpressures. The backpressure creates a resistance to the flow, resulting in lower inlet gas flow rates. Static culture maintains the chamber pressure at atmospheric levels and so gas flow rates are much higher.

Several steps were taken to minimize evaporation of the medium. The gas mixture was bubbled through a heated humidifier before it entered the culture chamber. The chamber was sealed with Teflon tape, allowing gas to exit, but trapping moisture. The gas flow rate was also set as low as the regulator would allow. These conditions worked well for 9.5-dpc embryos. For the earlier embryos, it was necessary to take additional steps to minimize evaporation. This was achieved by placing a thin layer of mineral oil over the medium. This layer helped maintain $\mathrm{pH}$ at normal levels (7.0 to 7.2). Under these conditions, the percentage of embryos that turned rose from $10.5 \%(n=38)$ to $41.4 \%(n=82)($ Table 3$)$. The yolk sacs of the embryos cultured with mineral oil were smooth and circulation in the yolk sac was constant. Heart rates of the embryos cultured in mineral oil were also more in line with previously published rates for freshly dissected embryos (Porter \& Rivkees, 2001).

\section{Development of 8.5-dpc embryos in culture}

During normal embryogenesis, in the 24 hours between $8.5-\mathrm{dpc}$ and 9.5 -dpc, many important developmental changes occur. Most notably, the heart begins to beat and the embryos turns, becoming physically separated from the yolk sac. These milestones were recorded to assess normal growth. Embryos grown for 24 hours in culture (Figure 2, a-b) were compared to freshly dissected 9.5-dpc embryos (Figure 2, c). The cultured embryos were found to match freshly dissected embryos both in size and appearance. 
In all cultured embryos $(n=82)$, blood flow could readily be observed in the vascular channels of the yolk sac and embryo as well as in the heart. During culture, the heart rate increased from $92.2 \pm 21.7$ to $107.2 \pm 27.1 \mathrm{bpm}$, as stated in Table 3 . These rates do not vary significantly from rates published for freshly dissected embryos (Porter \& Rivkees, 2001). Even after 24 hours in culture, the embryos still had very strong heartbeats and good flow through their blood vessels. This can also be observed in several of the time-lapse movies presented in the subsequent section.

To assess the extent of embryonic turning, we compared our static culture technique with roller culture. Our culture conditions resulted in $41.4 \%$ of embryos turning $(n=82)$, as compared to $58.3 \%$ of embryos turning in roller culture $(n=19)$. Only embryos older than stage 9 (first heart fibrillations visible) were included in these statistics. It is thought that all embryos above stage 9 have the ability to turn in culture, although error could be introduced since it is difficult to predetermine an embryo's potential to turn. The average initial stage of the embryos was $13.1 \pm$ 2.6 for static culture and $12.9 \pm 3.0$ for roller culture. Turning has, in the past, been the largest impediment to static culture for embryos this age. The high rate at which embryos turned in static culture highlight the success of this technique.

\section{Development of 9.5-dpc embryos in culture}

Development of 9.5-dpc embryos is characterized by the onset of organogenesis, significant changes in embryonic size and the maturation of many of the head features. The size and morphological traits of cultured 9.5-dpc embryos (Figure 2, e) and freshly dissected 10.5-dpc embryos (Figure 2, f) are grossly indistinguishable. 
During normal embryonic development, somites are added at a rate of approximately one pair every hour and a half (Hogan, et al., 1994). The somite addition rate can be taken as a "biological clock" and was monitored as a sign of embryonic health during culture. As seen in Figure 4, somite addition rates measured in culture were quite consistent with the somite addition rates reported for embryonic development in vivo (Copp \& Cockroft, 1990). After 24 hours, the tissue becomes quite opaque, possibly because of the initiation of tissue necrosis late in culture. As such, 9.5-dpc embryos should not be cultured past 18 hours.

Because heart rates appear to be very sensitive to small changes in the local environment, the heart rate was again used as an indicator of embryonic health. Heart rates are shown in Figure 3 for 9.5-dpc embryos cultures and are compared to previously published data (Porter \& Rivkees, 2001). The published rate for 9.5-dpc was measured in whole embryos, however the rate for 10-dpc embryos was measured in isolated hearts (Porter \& Rivkees, 2001). Embryo heart rates in static culture appeared to remain constant over the 24-hour incubation period. The difference between our rates and published rates after 24 hours, though not statistically significant, could be due to the comparison of whole embryo heart rates and those from isolated hearts. After 24 hours in culture, the standard deviation of the heart rates of the cultured embryos became quite large. Therefore, the culture period was never extended beyond 24 hours.

\section{Time-lapse movies}

Because this static embryo culture system was developed with the aim of using fluorescent or bright field imaging to observe mammalian development, several movies were made to demonstrate the success of the technique. These are provided here in panel form and as timelapse movies in the supplemental material that is presented on the Internet. 
In the first movie (Figure 5, also see the supplementary video 1), we show neural tube closure in the hindbrain region. The neural folds approximate in a caudal to rostral manner, as occurs in vivo. Interestingly, small, dynamic groups of cells are evident at the midline during closure, indicating that some cells may delaminate during this process.

Another major event in the development of $8.5-\mathrm{dpc}$ embryos is axial rotation or turning (Figure 6 , shown in the supplementary video 2). The embryo is clearly seen rotating its upper body away from the yolk sac. The body detaches from the yolk sac in a rostral-caudal direction, leaving only the caudal end attached. Clearly highlighted is the posterior closure of the ventral side. The head and heart disappear and then reappear below the caudal end of the embryo.

The addition of somites was followed (Figure 7, see also supplementary video 3) in 9.5-dpc embryos. Embryos typically start off with 12 somites and progressively add somites through the culture period. The somites are labeled as they are added using asterisks (Figure 7). The rate of somite addition matches that reported for freshly dissected embryos (Hogan, et al., 1994).

The last movie highlights the main strength of this technique, which is the ability to follow fluorescently labeled cell movements in tissues. The embryo shown carries a green fluorescent protein (GFP) reporter that is expressed specifically in primitive erythroblasts (Dyer, et al., 2001). This allows the direct observation of blood flow in the yolk sac and the embryo and reveals normal circulation (Figure 7, as well as supplementary video 4). Careful inspection also provides insight into early changes in vascular morphology. By including a marker such as a GFP, it is now possible to observe dynamic interactions in mammalian embryos that lead to the formation of differentiated tissues. 


\section{Discussion}

It has generally been assumed that the most significant limitation of static culture of postimplantation embryos is diffusion. We present results that clearly show that this problem is not as restrictive as was previously thought. This is especially highlighted by the high rate at which 8.5-dpc embryos were able to complete the turning process under static conditions and the constant rate of somite addition in the 9.5-dpc embryos. Though the ability to culture embryos of this stage under static conditions in incubators was reported previously (Wan, et al., 1982), the technique had never been expanded to direct microscopic observation. With this goal in mind, we have optimized environmental conditions for normal embryonic development on the microscope stage.

It is not solely the diffusion limitation of static culture that prevents proper development on microscopic stages, but the greater difficulties in controlling environmental conditions. In roller culture, high pressure reduces gas flow rates to very low levels, minimizing evaporation. Similarly, in tissue incubator cultures, the extremely humid environment minimizes evaporation and concentration of the medium. Since it is not feasible to reach a humidity level similar to tissue incubators during culture on the microscope stage, it was necessary to take further steps to prevent evaporation, through the addition of a very thin layer of mineral oil.

The ability to culture mouse embryos within a chamber accessible to microscopy overcomes the limitations of visualizing the cellular dynamics of mouse development. While the technique is limited in providing an 18-24 hour window on development, it improves upon roller culture in that embryos can be continuously observed over this period. With the growing number of transgenic mouse lines expressing fluorescent marker proteins, it should be possible to observe the localization of any gene product and to track the movement and development of cells expressing that gene. 
We are working to extend this technique in two ways. First, preliminary results using 7.5-dpc embryos ( $n=9$, data not shown) show promise that younger embryos too can develop normally in static culture. Second, work is also underway using the technique to observe cellular development in mutant strains. Through time-lapse imaging, we should be able to bridge the gap between gene function and the subsequent changes in morphology.

\section{Materials and Methods}

\section{Dissection}

Male breeder mice were mated with CD-1 females overnight. The presence of a vaginal plug was taken as 0.5-dpc. Embryos were collected on the morning of the eight or ninth day as noted in a plexiglass hood heated to $37^{\circ} \mathrm{C}$ with a chicken incubator heater (Lyon Electric Company \# 115-20). Dissecting outside of the heated hood causes decreased viability. Dissecting medium was prepared by mixing $45 \mathrm{ml}$ of D-MEM/F-12 (Gibco \# 11330032) with 4.5 $\mathrm{ml}$ of heat-inactivated fetal bovine serum (Gibco \# 16140063) and $0.5 \mathrm{ml}$ of pen-strep solution (Irvine Scientific \# 9366). The dissecting medium was warmed to $37^{\circ} \mathrm{C}$ prior to dissection. Females were euthanized with $\mathrm{CO}_{2}$ followed by cervical dislocation. The uterine horns were dissected out and placed in warmed dissecting medium and the embryos isolated. Yolk sacs were left intact on 8.5-dpc embryos but were removed for 9.5-dpc embryos. For the imaging of neural tube closure, the yolk sac of 8.5-dpc embryo was removed.

\section{Rat Serum}

Though several sources of commercial rat serum were investigated, it was found that the best cultures came from serum that we made ourselves. Both the blood collection site and the JONES 
anesthetic in the commercial sources were presumed to be the cause of the lower quality serum. In particular, the use of ether as the anesthetic is likely to be essential because it can be completely eliminated by aeration (Sturm \& Tam, 1993).

Rat serum was prepared from blood collected from the dorsal aorta of male rats (Copp \& Cockroft, 1990) using the following modifications. Blood was collected into vacutainer tubes (Becton-Dickinson \# 366512) using a butterfly needle (Becton-Dickinson \# 367283). After collection, the blood was centrifuged at $2500 \mathrm{rpm}$ for 20 minutes. The plasma was isolated and supernatant from multiple rats were pooled. The serum was then centrifuged again at $2500 \mathrm{rpm}$ for 10 minutes to remove remaining cells. The supernatant was heat-inactivated at $56^{\circ} \mathrm{C}$ for 30 minutes with the lid removed to allow the ether to evaporate. The serum was then filtered using a $0.45 \mu \mathrm{m}$ filter and aliquoted into $1 \mathrm{~mL}$ samples. These samples were stored in $\mathrm{a}-80^{\circ} \mathrm{C}$ freezer.

\section{Incubation and Culturing of Embryos}

The culturing medium consisted of $1 \mathrm{~mL}$ D-MEM/F12, $1 \mathrm{~mL}$ heat-inactivated rat serum, $20 \mu \mathrm{L}$ Pen-Strep and $20 \mu \mathrm{L}$ HEPES buffer solution $1 \mathrm{M}$ (Irvine Scientific, Cat No. 9319). The medium was sterile filtered with a $0.2 \mu \mathrm{m}$ filter and allowed to equilibrate, with the lid off, in a $\mathrm{CO}_{2}$ incubator for 1 hour. The dissected embryos were then transferred to culture chambers (Nunc Lab-tek, 2 chambers/ coverglass, Cat No. 155380) with a minimal amount of the dissecting media using a transfer pipette. Three embryos were placed per chamber and $2 \mathrm{~mL}$ of the culturing media was added. For the $8.5-\mathrm{dpc}$ embryos, the medium was then covered with a very thin layer of mineral oil (Sigma \# M8410).

After transfer to the chamber slides, the embryos were pre-incubated for one hour at $37^{\circ} \mathrm{C}, 5 \%$ $\mathrm{CO}_{2}$ in a tissue incubator and then still-photographed at 2.5x magnification. Initial heart rate 
and somite counts were made and the $\mathrm{pH}$ of the media was measured by removing a drop of medium. The pre-equilibration in a $\mathrm{CO}_{2}$ incubator reliably adjusted the initial $\mathrm{pH}$ to 7.2.

In order to properly aerate the chambers, a hole was made in the side of the Lab-Tek chamber lid using a soldering iron. Inlet gas $\left(5 \% \mathrm{CO}_{2}\right.$, balance air) was passed through a bubbler to humidify the air. The bubbler was custom built by the Caltech Glass Shop, however most commercially available gas-washing bottles can be used for this purpose. The flow rate was set as low as the regulator could operate. The chamber was sealed using Teflon tape.

The bubbler, culture chamber and a dissecting microscope were all placed within a heater box that was constructed around the microscope from cardboard ( $4 \mathrm{~mm}$ thick) covered with thermal insulation (Reflectix Co.; 5/16" thick, foil-foil insulation). The temperature was set to $37^{\circ} \mathrm{C}$ using an egg-incubator heater (Lyon Electric Company \# 115-20) and allowed to warm for several hours before culture to prevent thermal drift of the microscope components. This arrangement is shown in Figure 1.

Static images of the embryos were taken at 6 hours, 12 hours and 24 hours. The heart rate and somite numbers were counted visually at these intervals. The intermediate measurements were found to harm the 8.5-dpc embryos and were eliminated in later studies. For the 8.5-dpc embryos, initial and final heart rates were measured and the extent of development was assessed by head fold closure, maturation of the vasculature and ability to turn. After 24 hours, the embryos were fixed in $4 \%$ paraformaldehyde overnight at $4^{\circ} \mathrm{C}$ and then transferred to PBS $\left(\mathrm{Ca}^{2+}\right.$ and $\mathrm{Mg}^{+}$free $)$and stored at $4{ }^{\circ} \mathrm{C}$.

\section{Time-lapse Imaging}

In order to image the $8.5-\mathrm{dpc}$ embryos, it was necessary to immobilize the embryos. This was done by tying a hair around the deciduas and resting the ends of the hair on the bottom of the JONES 
chamber. Older embryos were more stable in the culture dish and therefore were not immobilized.

The embryos were imaged with either bright-field or confocal microscopy. Single images were taken every five minutes. The bright-field images were taken on an inverted microscope (Zeiss Axiovert) using a SIT camera (Hamamatsu) and acquired using VIDIM (VIDeo IMaging) software written by Scott Fraser, Jes Stollberg and Gary Belford for Imaging Technology Incorporated Series 151 image processors. The confocal images were taken on two distinct inverted confocal microscopes (BioRad MRC600; Zeiss LSM PASCAL). All images were taken at $5 \mathrm{X}$ magnification, except for the somite addition time-lapse, which is at $2.5 \mathrm{X}$ magnification. 


\section{References}

Bulic-Jakus F, Vlahovic M, Juric-Lekic G, Crnek-Kunstelj V, Serman D. 1999. Gastrulating rat embryo in a serum-free culture model: Changes of development caused by teratogen 5azacytidine. ATLA-Alternatives to Laboratory Animals 27:925-933.

Copp AJ, Cockroft DL. 1990. Postimplantation mammalian embryos: a practical approach. Oxford ; New York: IRL Press. 357 p.

Dyer MA, Farrington SM, Mohn D, Munday JR, Baron MH. 2001. Indian hedgehog activates hematopoiesis and vasculogenesis and can respecify prospective neurectodermal cell fate in the mouse embryo. Development 128:1717-1730.

Eto K, Osumi-Yamashita N. 1995. Whole embryo culture and the study of postimplantation mammalian development. Develop Growth Differ 37:123-132.

Hogan B, Beddington R, Costantini F, Lacy E. 1994. Manipulating the mouse embryo : a laboratory manual. Plainview, N.Y.: Cold Spring Harbor Laboratory Press. 497 p.

Janzen RG, Andrews GK, Tamaoki T. 1982. Synthesis of secretory proteins in developing mouse yolk sac. Dev Biol 90:18-23.

Katoh M, Kimura R, Shoji R. 1998. Embryogenesis-promoting factors in rat serum. J Exp Zool $281: 188-200$.

Martin P, Cockroft DL. 1999. Culture of postimplantation mouse embryos. Methods Mol Biol 97:7-22.

Porter GA, Jr., Rivkees SA. 2001. Ontogeny of humoral heart rate regulation in the embryonic mouse. Am J Physiol Regul Integr Comp Physiol 281:R401-407. 
Sturm K, Tam PPL. 1993. Isolation and culture of whole postimplantation embryos and germ layer derivatives. Methods Enzymol 225:164-190.

Tam PP. 1998. Postimplantation mouse development: whole embryo culture and micromanipulation. Int J Dev Biol 42:895-902.

Wan YJ, Wu TC, Damjanov I. 1982. Development of early somitic mouse embryos in static culture in vitro. J Exp Zool 220:219-225. 


\section{Acknowledgements}

The authors wish to acknowledge Carole Lu for help with the initial cultures, and Joaquin Gutierrez and Deanna Mohn for technical assistance. Research by the authors was supported by grants from the American Heart Association for D.C., from the National Institutes of Health to M.H.B., from the Human Frontiers S P grant to S.E.F. We would also like to thank Powell foundation for the partial support of this work through the option of bioengineering at Caltech.

Correspondence and requests for materials should be addressed to Mary Dickinson (maryd@gg.caltech.edu). 


\section{Tables}

Table 1 - Summary of staging system used for 8.5 dpc embryos

Characteristic of Embryonic Development

Stage

Formation of neural plate

Appearance of first somite

Formation of linear heart tube

First heart beats

Commencement of head fold closure

Heart begins looping

Fusion of head folds

Beginning of axial rotation

Axial rotation $50 \%$ complete

Completion of axial rotation

1

4

5

10

11

12

15

17

19

22

Table 2 - Comparison of Rat Serum Collection Techniques

\begin{tabular}{|c|c|c|c|c|c|}
\hline Supplier & Anesthetic & Source & Sex & $\begin{array}{c}\text { No. of Somites } \\
\text { After Culture of } \\
\text { 9.5-dpc }\end{array}$ & $\begin{array}{l}\text { Percent } \\
\text { Turning at } \\
\text { 8.5-dpc }\end{array}$ \\
\hline Gibco (Carlsbad, CA) & $\mathrm{CO}_{2}$ & $\begin{array}{l}\text { Jugular } \\
\text { Bleed }\end{array}$ & $\begin{array}{l}\text { Mixed } \\
\text { sex }\end{array}$ & $20.0 \pm 2.4$ & $\mathrm{~N} / \mathrm{A}$ \\
\hline $\begin{array}{l}\text { Seracare, Inc. } \\
\text { (Oceanside, CA) }\end{array}$ & $\mathrm{CO}_{2}$ & $\begin{array}{l}\text { Cardiac } \\
\text { collection }\end{array}$ & $\begin{array}{l}\text { Male } \\
\text { only }\end{array}$ & $22.2 \pm 4.0$ & $0 \%$ \\
\hline $\begin{array}{l}\text { Biochemed } \\
\text { Pharmacologicals, Inc. } \\
\text { (Winchester. VA) }\end{array}$ & $\mathrm{CO}_{2}$ & $\begin{array}{l}\text { Jugular } \\
\text { Bleed }\end{array}$ & $\begin{array}{l}\text { Mixed } \\
\text { sex }\end{array}$ & $23.2 \pm 2.0$ & $N / A$ \\
\hline Home-made serum & Ether & $\begin{array}{l}\text { Dorsal } \\
\text { Aorta }\end{array}$ & $\begin{array}{l}\text { Male } \\
\text { only }\end{array}$ & $21.3 \pm 3.7$ & $10.5 \%$ \\
\hline
\end{tabular}


Table 3 - Results from Culture With and Without Mineral Oil Addition

\begin{tabular}{|c|c|c|c|c|}
\hline & $\begin{array}{l}\text { Percent } \\
\text { Turning }\end{array}$ & $\begin{array}{l}\text { Initial Heart Rate } \\
\text { (bpm) }\end{array}$ & $\begin{array}{c}\text { Final Heart Rate } \\
(\mathrm{bpm})\end{array}$ & $\mathrm{n}=$ \\
\hline $\begin{array}{l}\text { Static Culture without } \\
\text { Mineral Oil }\end{array}$ & 10.5 & $91.2 \pm 26.5$ & $60.3 \pm 37.0$ & 38 \\
\hline $\begin{array}{l}\text { Static Culture with } \\
\text { Mineral Oil }\end{array}$ & 41.4 & $92.2 \pm 21.7$ & $107.23 \pm 27.09$ & 82 \\
\hline $\begin{array}{l}\text { Freshly } \quad \text { Dissected } \\
\text { Embryos (Porter et } \\
\text { Rivkees, 2001) }\end{array}$ & 100 & $80 \pm 2$ & $91 \pm 3$ & $\mathrm{~N} / \mathrm{A}$ \\
\hline
\end{tabular}




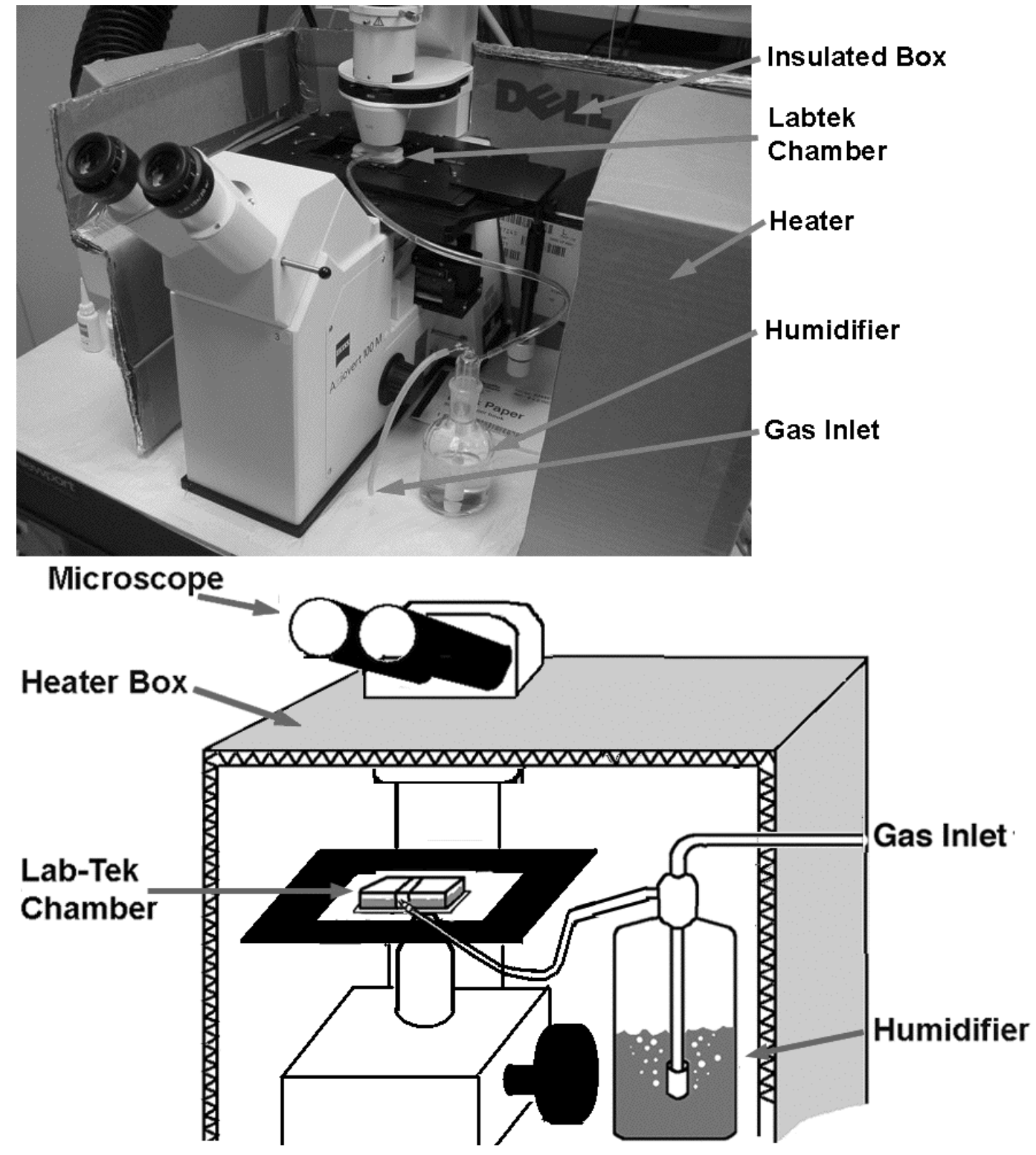

JONES 


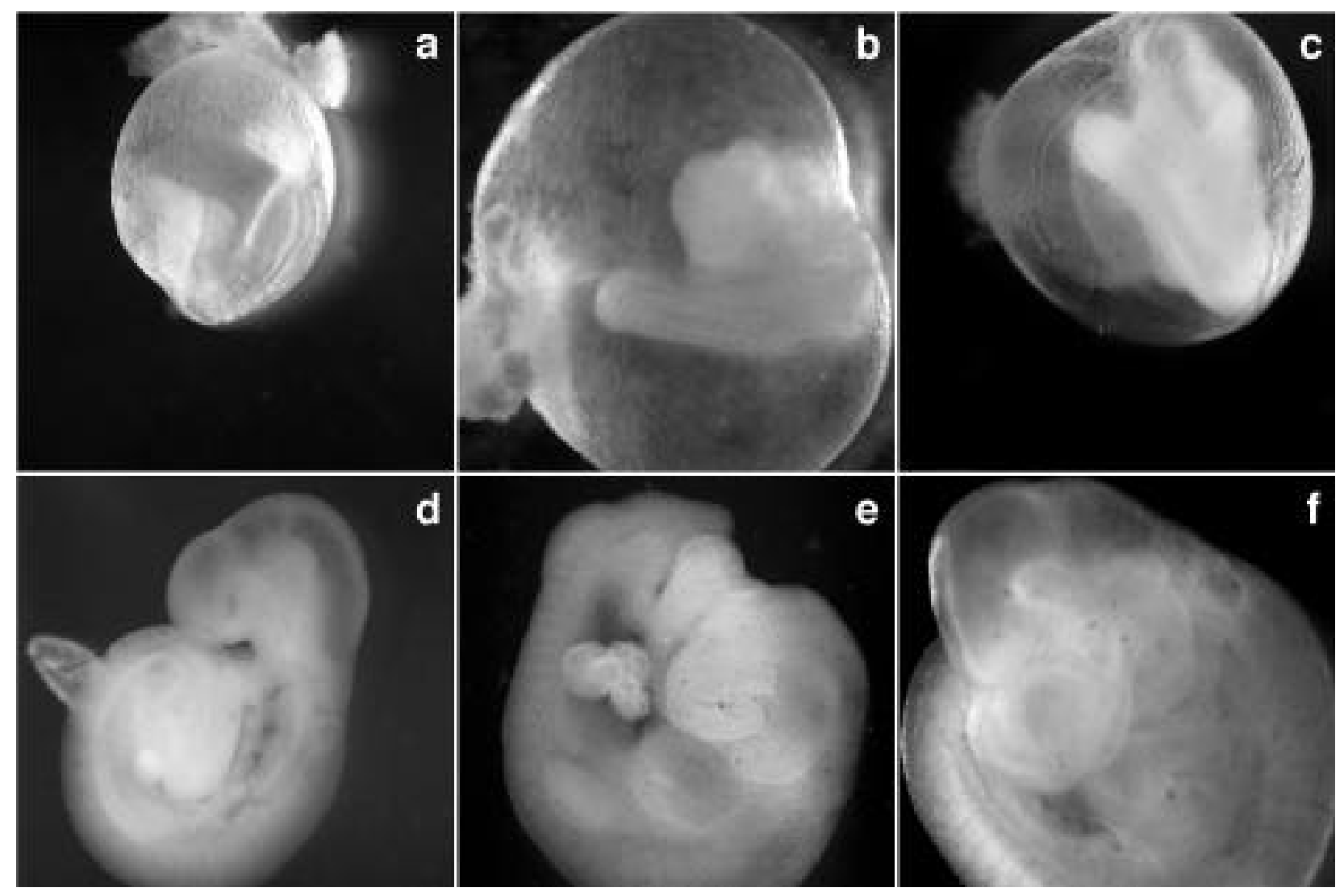

JONES 


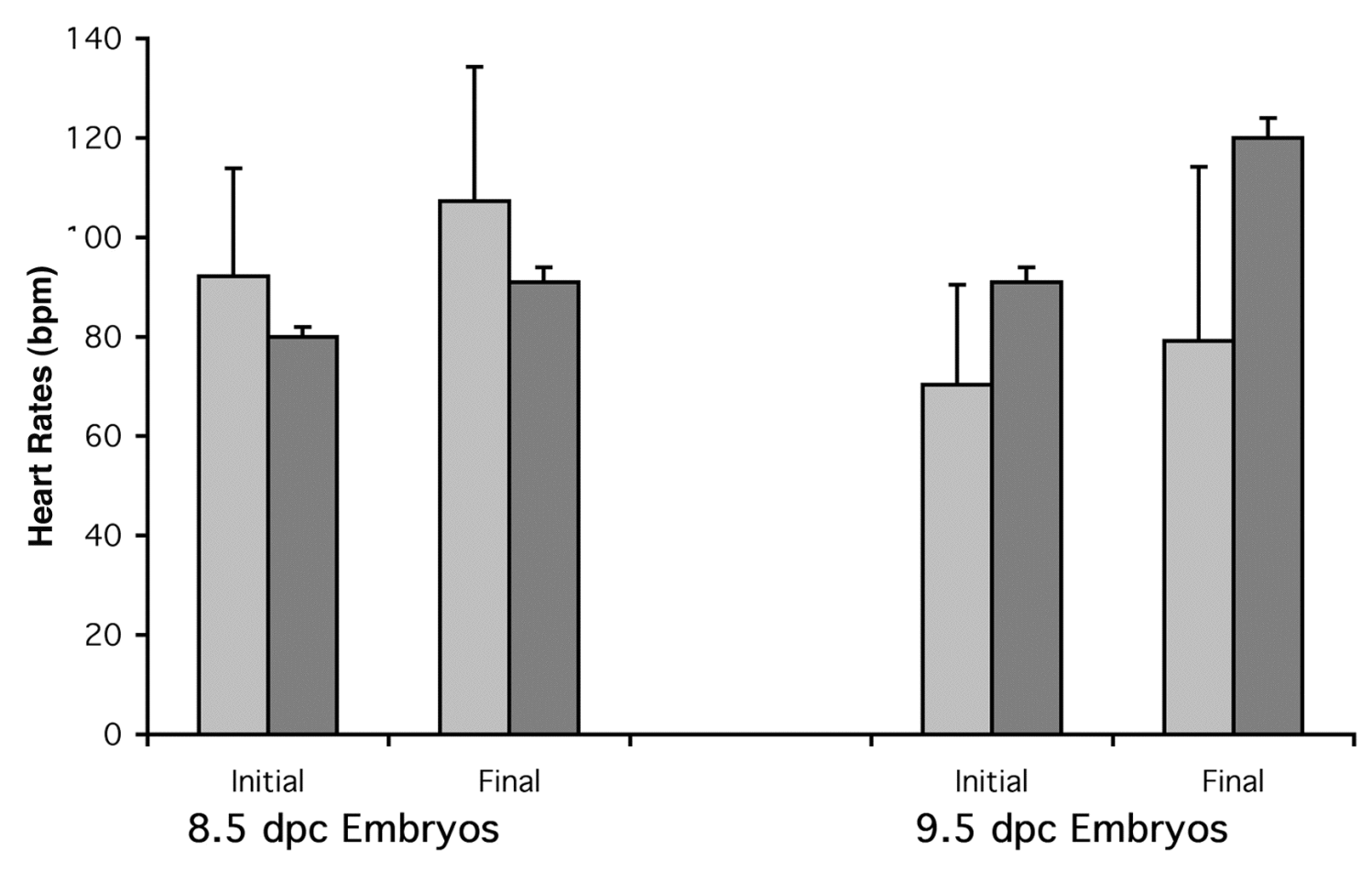

$\square$ Static $\square$ Freshly Dissected by Porter, et al.

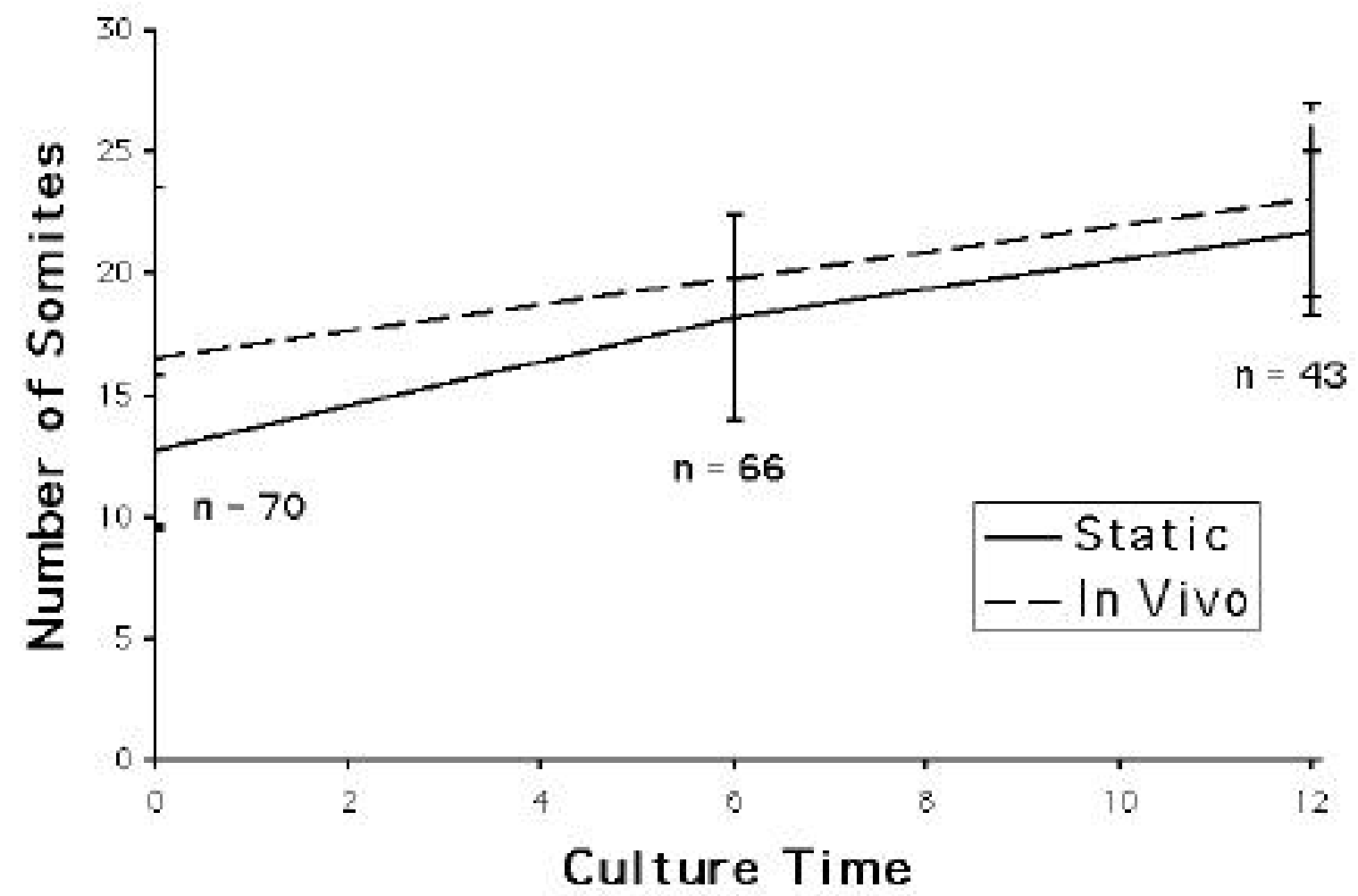

JONES 

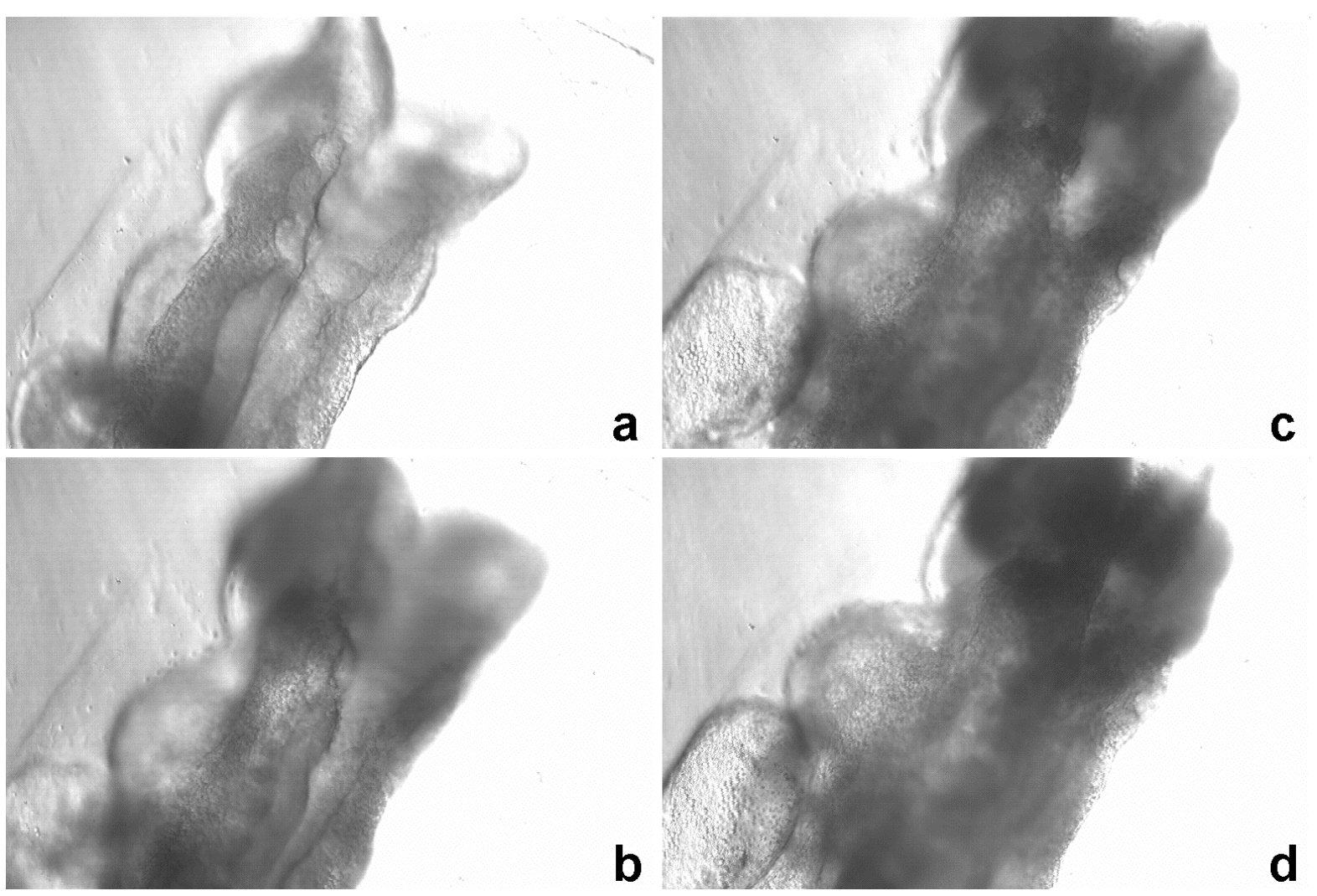

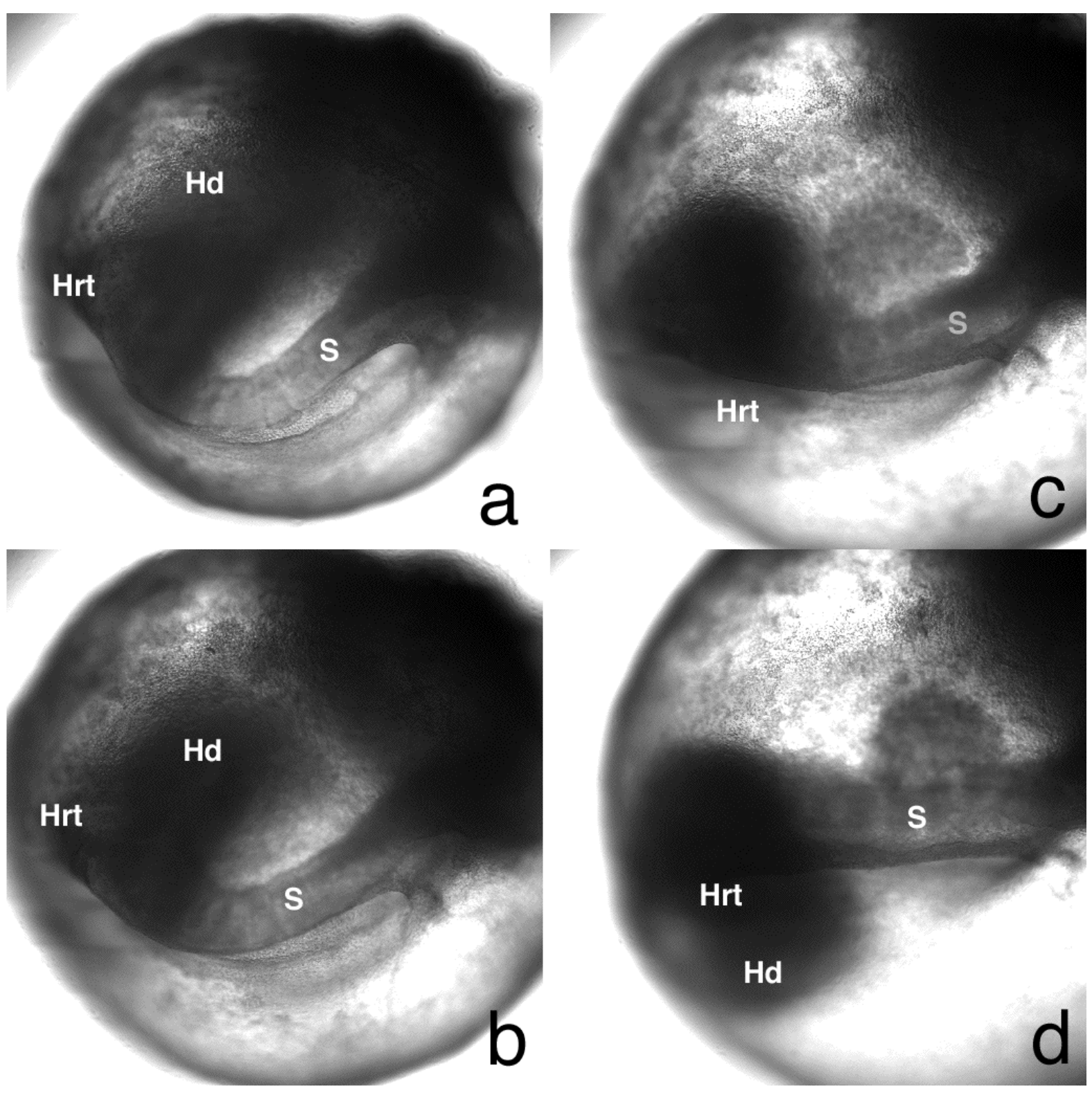

Hrt

C

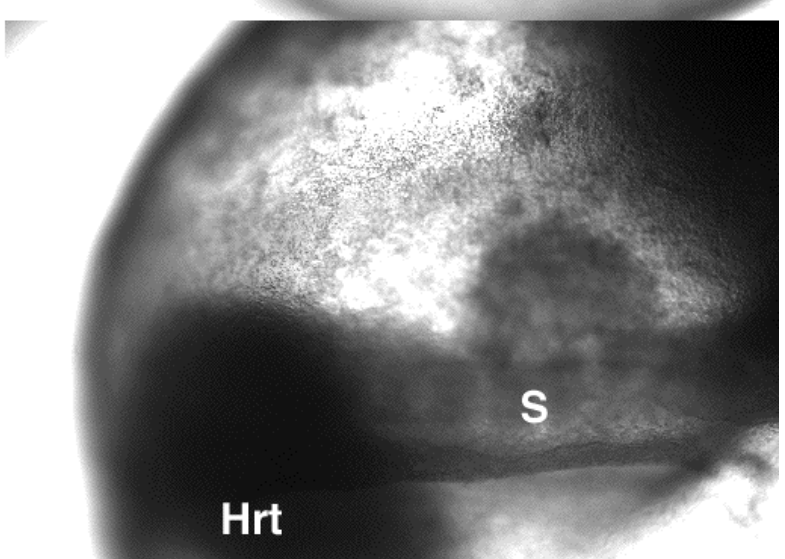

Hd

d 

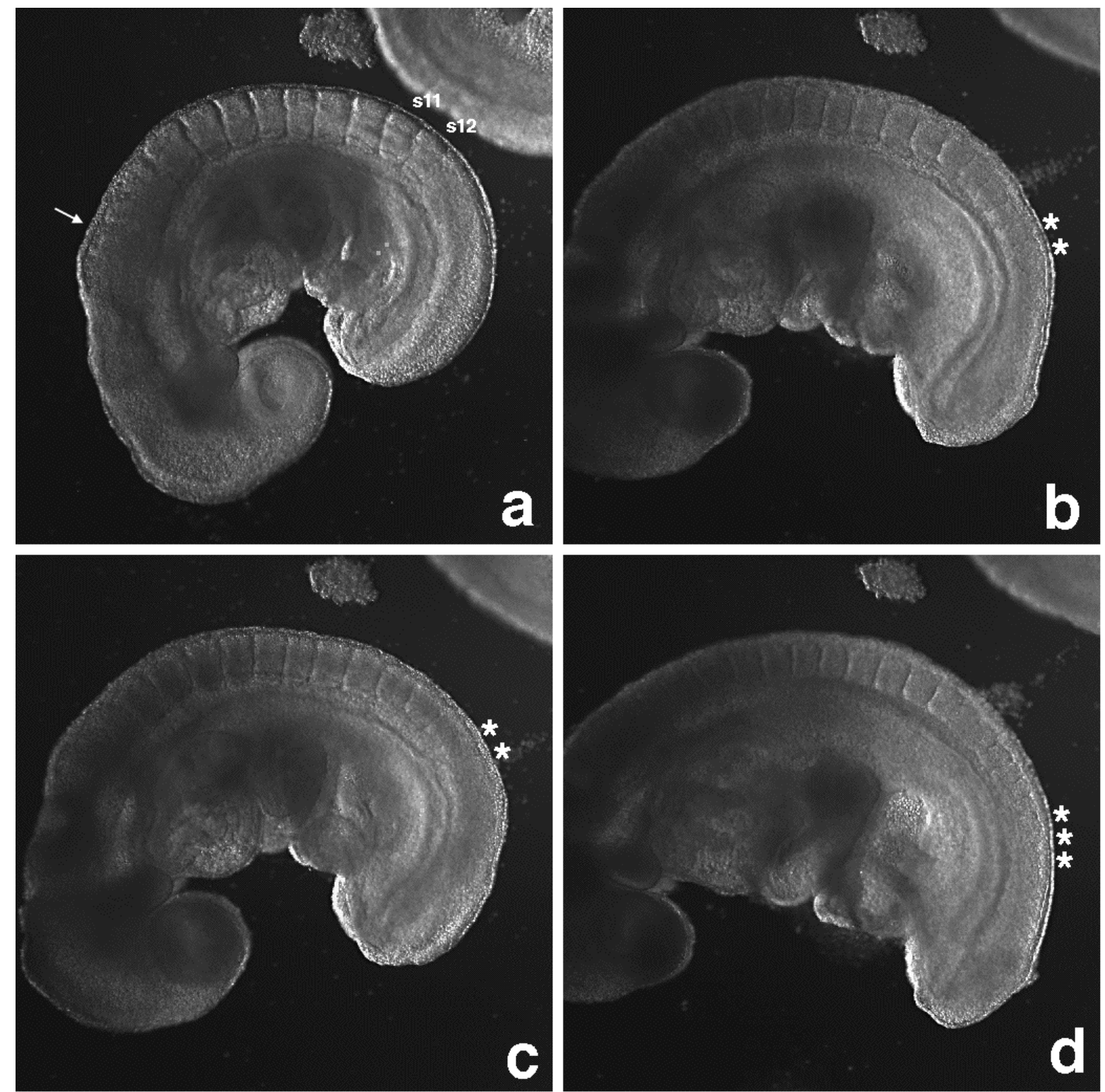


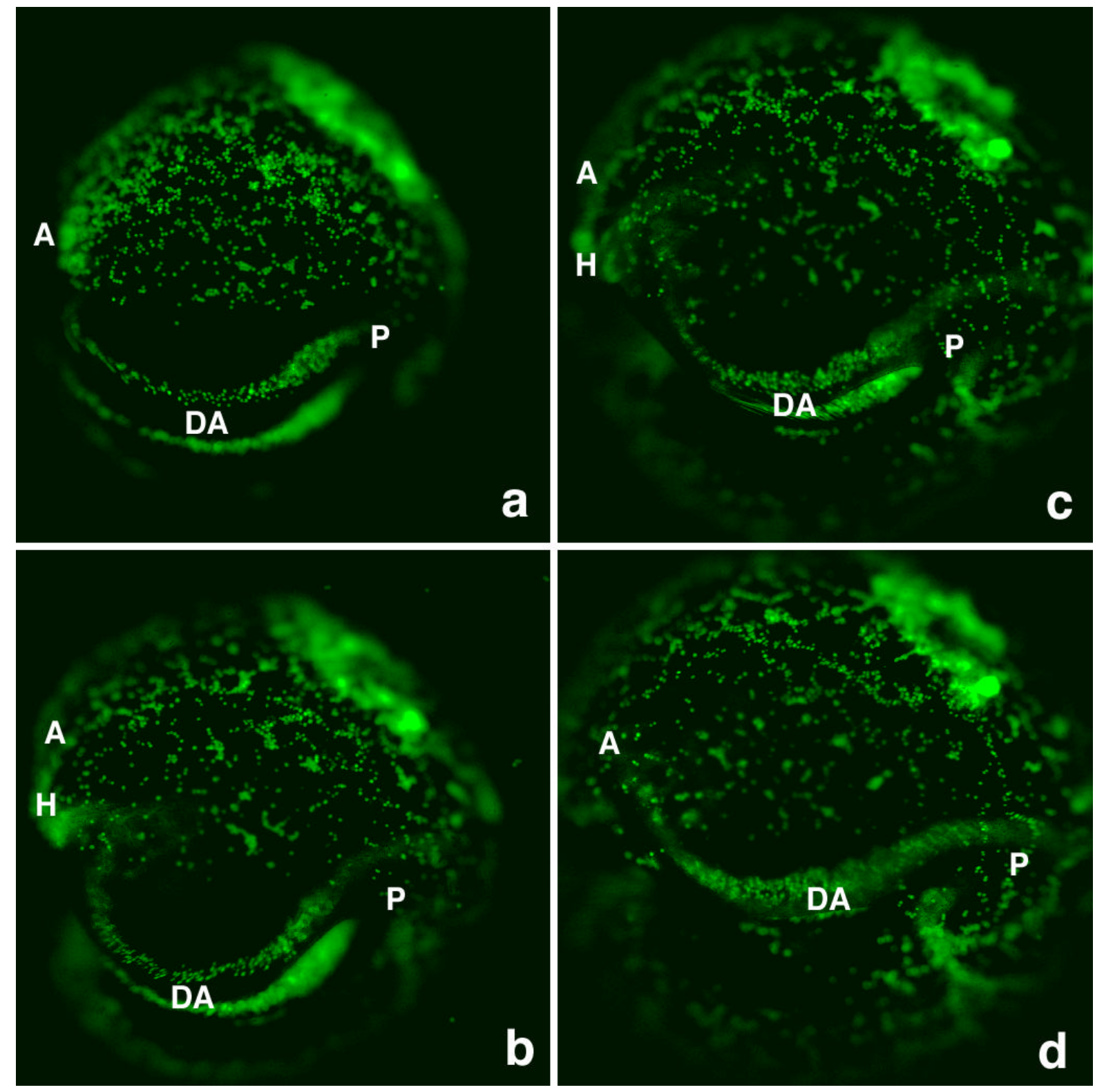

JONES 
Figure 1. Experimental System. Modified Labtek chambers were placed on the microscope stage. The chambers were sealed with Teflon to prevent the evaporation of media. The gas mixture $\left(5 \% \mathrm{CO}_{2}\right.$, balance air) was humidified before flowing over the media. The entire setup was contained within an insulated box and heated to $37^{\circ} \mathrm{C}$. The actual arrangement of the heater box (with top and side panels missing) is shown in frame A, as well as a schematic of the system in panel $B$.

Figure 2. Embryo Culture Comparison with Embryo Development In Vivo. 8.5-dpc (a) and 9.5dpc (d) embryos before culture and after 24 hours in static culture (b \& e), which are compared with embryos that have developed for an equivalent period in vivo from 8.5-dpc (c) and 9.5-dpc (f). Magnification is $4 \mathrm{X}$ for a-c, and $2.5 \mathrm{X}$ for frames $\mathrm{d}-\mathrm{f}$.

Figure 3. Heart Rates During Culture. Heart rates were followed as indicators of embryonic health and compared with data from Porter \& Rivkees, 2001. The left hand columns, which represent the 8.5-dpc embryos before and after culture (light gray), are compared with freshly dissected embryos (dark gray). On the right hand side, columns represent the heart rate before and after culture for 9.5-dpc embryos (light gray) that are compared with freshly dissected embryos from the same age (dark gray).

Figure 4. Comparison of Somite Addition During Culture and In Vivo. The number of somites of 9.5-dpc embryos in culture were counted (solid line) at $t=0,6$ and 12 hours and compared with the in vivo rates (dashed line). A final somite count at 24 hours was not performed because the embryos had become quite opaque and it was difficult to count somites accurately.

Figure 5. Time-lapse of neural tube closure in the hindbrain region. Panels illustrate hindbrain fusion in an 8.5-dpc embryo. The yolk sac was removed for this movie. The time-lapse covers a 14-hour period of time. The associated movie was imaged at a rate of one frame every 5 minutes and the panels present every $45^{\text {th }}$ frame. 
Figure 6. Time-lapse of embryonic turning. The time-lapse covers an 11-hour period of time. In this time, the embryo goes from a being u-shaped to rotating into "fetal position". In the process, the embryo detaches from the yolk sac. The head $(\mathrm{Hd})$, heart (Hrt) and somites $(\mathrm{S})$ are labeled on the diagram. The imaging rate was one frame every 5 minutes, and the panels present every $40^{\text {th }}$ frame.

Figure 7. Time-lapse of somite addition. Frames illustrate somite addition in a 9.5-dpc embryo. The time-lapse covers a 13.5-hour period of time. The asterisk $\left(^{*}\right)$ marks new somites that have been added since the last panel. The addition of the somites is better seen in the accompanying time-lapse movie. The images in the movie were taken every five minutes, and the panels represent every $50^{\text {th }}$ frame.

Figure 8. Blood Circulation in an 8.5-dpc Cultured Embryo. Panels illustrate the circulating red blood cells (in green) in an 8.5-dpc embryo that expresses GFP driven by the $\varepsilon$-globin promoter. (Dyer, et al., 2001) The time-lapse covers a 12.5-hour period of time during which the embryo is undergoing turning. The location of the anterior $(A)$ and posterior $(P)$ extremities of the embryo has been noted, as well as the dorsal aorta (DA) and the heart $(H)$ when visible. The images were taken every 5 minutes, and the panels represent every $50^{\text {th }}$ frame from the movie. 